and statistically significant; though none of the patient demographic features or serological markers were found to be associated with the delta change in phosphate to predict high risk patients. The median recovery time of 14 days is less than what is reported in adult reviews. FCM infusions need preassessment, counselling and post infusion monitoring to assess effectiveness and recovery.

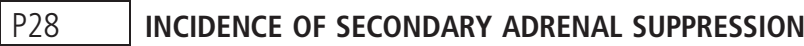 AFTER PROLONGED USE OF GLUCOCORTICOID THERAPY FOR CHILDREN WITH INFLAMMATORY BOWEL DISEASE}

${ }^{1}$ Virginia Chatzidaki, ${ }^{2}$ Rebecca Renji Chungath, 'Sarang Tamhne, 'Marcus Auth, ${ }^{1}$ Fiona Cameron, 'Manjula Velayudhan Nair, ${ }^{1}$ Jeng Haw Cheng, 'Stephen Allen, ${ }^{1}$ Elizabeth Renji, ${ }^{1}$ Jo Blair. ${ }^{1}$ Alder Hey Children's Hospital Trust; ${ }^{2}$ Newcastle medical school

\subsection{6/flgastro-2021-bspghan.38}

Introduction Glucocorticoids (GCs) are used in all forms of paediatric inflammatory bowel disease (IBD) for their antiinflammatory and immunosuppressive effect. Prolonged GC treatment may suppress the hypothalamic-pituitary-adrenal (HPA) axis causing secondary adrenal suppression (SAS), despite using a weaning regime, necessitating the use of hydrocortisone for replacement. The incidence and severity of SAS is poorly predictable and may be related to both the cumulative dose of GCs, but also to individual factors. Standard and low dose short Synacthen tests (SST, LDSST) are used to assess recovery of the HPA axis following a prolonged course of steroids.

Aims To report:

1. the incidence of SAS following GC treatment for IBD in our centre

2. the time to HPA axis recovery in patients with SAS

3. risk factors for SAS

Patients and Methods 33 children with IBD (19M, 10-18 years) previously treated with GCs, who had been investigated for SAS from 01/01/2017 and 30/10/2020 were identified. Baseline information including age, sex, somatometric parameters, IBD diagnosis, age at diagnosis and maintenance treatment were collected. All their GC courses $(n=47)$ in the defined period were reviewed, and those that led to SAS were compared for length and GC doses, previous exposure to GC, tests for HPA axis recovery and the requirement for hydrocortisone replacement therapy.

Results Of the 33 children, $15 / 33(45.5 \%)$ had ulcerative colitis (UC), 10/33 (30.3\%) had Crohn's Disease (CD) and 8/33 (24.2\%) were unclassified (IBDU). 10 children that were tested using non-standard tests for adrenal suppression were excluded.

23 children had their HPA axis recovery tested after 24 GC courses with LDSST $(19 / 24,79.2 \%)$ or SST $(5 / 24$, 20.8\%). 16/23 children had steroid courses of $\geq 12$ weeks, and $7 / 23$ had a shorter course ( $<11$ weeks) but had extended courses in the past (4/7) and/or high dose IV steroid (3/7). $14 / 23(60.9 \%)$ children had suboptimal response with peak cortisol range $129-441 \mathrm{nmol} / \mathrm{L}$ (table 1). 8/14 retests were available in the study period, 5/8(62.5\%) children recovered HPA axis function in a mean period of $6.83 \pm$ SD2.4 months and $3 / 8$ remained suppressed after a mean period of 4.6 \pm SD2.7 months.
Abstract P28 Table 1 Patients with inflammatory bowel disease tested with SSTs

\begin{tabular}{|c|c|c|c|}
\hline Characteristics & $\begin{array}{l}\text { Adrenal } \\
\text { Insufficiency } \\
(n=14)\end{array}$ & $\begin{array}{l}\text { Normal } \\
\text { Response } \\
(n=9)\end{array}$ & $\begin{array}{l}\mathrm{p}- \\
\text { value }\end{array}$ \\
\hline Age of Diagnosis, mean (range in yrs) & $11.7(6-15)$ & $11.6(9-15)$ & 0.29 \\
\hline \multicolumn{4}{|l|}{ Gender, n (\%) } \\
\hline - Male & $7(50 \%)$ & $5(55.6 \%)$ & 0.56 \\
\hline - Female & $7(50 \%)$ & $4(44.4 \%)$ & \\
\hline \multicolumn{4}{|l|}{ IBD phenotype, n(\%) } \\
\hline - Crohn's Disease & $4(28.6 \%)$ & $2(22.2 \%)$ & 0.56 \\
\hline - Ulcerative Colitis & $8(57.1 \%)$ & $5(55.6 \%)$ & 0.63 \\
\hline - IBD unclassified & $2(14.3 \%)$ & $2(22.2 \%)$ & 0.52 \\
\hline \multicolumn{4}{|l|}{ Other non-systemic GCs } \\
\hline - Rectal GCs & $5(35.7 \%)$ & $4(44.4 \%)$ & 0.50 \\
\hline - Inhaled CS (fluticasone) & 0 & $1(11.1 \%)$ & \\
\hline Total GCs courses, mean (range) & $1.79(1-3)$ & $2.33(1-4)$ & 0.10 \\
\hline \multicolumn{4}{|l|}{ Indication for test $\mathrm{n}(\%)$} \\
\hline - $\mathrm{GCs}$ course $\geq 12$ weeks & $11(78.6 \%)$ & $6(66.7 \%)$ & 0.43 \\
\hline - Previous GCs course $\geq 12$ weeks & $5(35.7 \%)$ & $7(77.8 \%)$ & 0.06 \\
\hline $\begin{array}{l}\text { - Use of } 10-20 \mathrm{mg} / \mathrm{kg} \text { (high dose) IV } \\
\text { Methylprednisolone }\end{array}$ & $5(35.7 \%)$ & 0 & \\
\hline Serum Basal Cortisol, mean (range) (nmol/L) & $177.6(<50-364)$ & $246.6(131-406)$ & 0.05 \\
\hline
\end{tabular}

The mean duration of courses that induced SAS was 154 86.8 days compared to $131.6 \pm 63$ days in those without SAS, $\mathrm{t}=0.44, \mathrm{p}=0.33$, and the previous days of exposure were 219 \pm 95 days for those with SAS compared 316.5 \pm 154.2 days for those without SAS, $\mathrm{t}=-1.2, \mathrm{p}=0.13$.

High dose IV methylprednisolone (10-20 mg/kg) was used in $5 / 24(20.8 \%)$ courses with $5 / 5(100 \%)$ and $3 / 5$ (60\%) inducing HPA axis suppression on the first testing and retesting respectively. Standard dose $(1.6 \mathrm{mg} / \mathrm{kg})$ IV methylprednisolone followed by oral weaning GC course was used in 9/24 (37.5\%) courses and 5/9 (55.6\%) induced HPA axis suppression. Standard dose oral prednisolone $(1-2 \mathrm{mg} / \mathrm{kg}$ ) was used in $10 /$ $24(41.7 \%)$ of the courses and 5/10 (50\%) induced SAS.

Conclusion SAS was detected in $60.9 \%$ of our IBD patients who were tested after GCs therapy. Our data support previous reports that additional factors to the duration of treatment with GCs, as the use of high dose of intravenous GCs, may influence the risk of SAS.

\section{P29 INDICATIONS OF PAEDIATRIC ENDOSCOPIES AND CORRELATION BETWEEN RESULTS AND CLINICAL OUTCOMES}

${ }^{1}$ Mara Popescu, ${ }^{1}$ Badra Farah, ${ }^{2}$ Mohamed Mutalib. 'King's College London; ${ }^{2}$ Evelina Children's Hospital, London

\subsection{6/flgastro-2021-bspghan.39}

Background Paediatric endoscopies are expensive and invasive procedures requiring general anaesthetic and should be performed to answer specific clinical questions. There is an increase in number of endoscopies performed in children but no epidemiological evidence of increasing paediatric GI disease burdens. Biopsies are also routinely performed during endoscopies adding to overall procedure cost.

Aims This study aimed to assess the indications of paediatric endoscopy, the association between endoscopic and histological 
results and the correlation between endoscopy and clinical outcome defined as discharge from hospital follow up.

Patients and Methods Retrospective review of clinical databases from June 2015 to July 2019. Only first diagnostic endoscopies were included, subsequent endoscopies and therapeutics endoscopies were excluded. Number of clinics prior and up to 6 months of endoscopies were reviewed and outcomes at 6 months were assessed. Correlation between endoscopy, histology results and outcome at 6 months were calculated using phi correlation

Results 196 children were included, $47.6 \%$ were females. Mean ( \pm SD) age 10.9 ( \pm 3.8). Indications were: abdominal pain $33 \%$, diarrhoea $14 \%$, rectal bleeding 9\%, suspected coeliac $7.5 \%$, constipation $9 \%$, reflux $12.3 \%$ and vomiting 15.1\%. $71.3 \%$ were upper endoscopies only and $28.7 \%$ were upper and lower endoscopies. $64 \%$ of all endoscopies were normal and $43.4 \%$ of the total were histologically normal. Number of clinics prior to endoscopies were $1.39( \pm 1.0)$ and children were seen $2.3( \pm 1.6)$ times in the six months after endoscopy. $18.5 \%$ of children were discharge from follow up within 6 months of having an endoscopy. There was weak (phi 0.18$)$ but statistically significant $(\mathrm{p}<0.05)$ correlation between endoscopy and discharge at 6 months. There was also weak (phi $0.2, \mathrm{p}$ 0.006) correlation between histology results and discharge at 6 months. There was a strong (phi $0.46 \mathrm{p}<0.005)$ positive correlation between endoscopic appearance and histological results.

All children were day cases and there was no complication identified in the studied population.

Conclusion Paediatric endoscopy appears to be a safe procedure with low risk of complication and most children were discharge on the same day. The majority of endoscopies in children were normal and about half were histologically normal with strong positive correlation between endoscopic and histological results, hence biopsies should not be performed if endoscopy is normal. Endoscopy did not appear to influence discharge from hospital follow up and the majority of children were still under follow up 6 months after having an endoscopy.

\section{P31 MINOR REFORMULATIONS OF INFANT FOOD FOR SPECIAL MEDICAL PURPOSES DUE TO EU REGULATIONS 2016/127 \& 2016/128 ARE WELL TOLERATED, ACCEPTED, COMPLIED WITH AND CONTINUE TO SUPPORT GROWTH IN INFANTS \& CHILDREN REQUIRING NUTRITION SUPPORT}

\footnotetext{
${ }^{1}$ Kiranjit Atwal, ${ }^{1}$ Gary Hubbard, ${ }^{2}$ Sarah Trace, ${ }^{3}$ Joanne Bartleman, ${ }^{3}$ Lucy Upton, ${ }^{4}$ Jo Pena, ${ }^{5}$ Jo Rayner, ${ }^{6}$ Sophie Aubrey, ${ }^{7}$ Victoria Steel, ${ }^{8}$ Jo Wildgoose, ${ }^{9}$ Kathleen Ross, ${ }^{10}$ Barbara Cochrane, ${ }^{3}$ Steve Kitchen, ${ }^{2}$ Heather Norris, ${ }^{11}$ Kirsty Jeffreys, ${ }^{11}$ Cathryn Tong, ${ }^{2}$ Rebecca Newbury, ${ }^{2}$ Laura Sealy, ${ }^{2}$ Abigail Robotham, ${ }^{2}$ Lynsey Francey, ${ }^{2}$ Lauren McVeigh, ${ }^{4}$ Imogen Clarke, ${ }^{1}$ Emily Wong, ${ }^{1}$ Benjamin Green, ${ }^{1}$ Elysia Hockley, ${ }^{1}$ Sean McGlinchey, ${ }^{1,13}$ Rebecca Stratton. ${ }^{1}$ Nutricia Ltd, Trowbridge, UK; ${ }^{2}$ University Hospitals Bristol and Weston NHS Foundation Trust, Bristol, UK; ${ }^{3}$ Birmingham Women's and Children's NHS Foundation Trust, Birmingham, UK; ${ }^{4}$ Newcastle upon Tyne Hospitals NHS Foundation Trust, Newcastle, UK; ${ }^{5}$ Bedfordshire Hospitals NHS Foundation Trust, Bedford, UK; ${ }^{6}$ Barts Health NHS Trust, London UK; ${ }^{7}$ South Tees Hospitals NHS Foundation Trust, Middlesbrough, UK; ${ }^{8}$ Bradford Teaching Hospitals NHS Foundation Trust, Bradford, UK; ${ }^{9}$ NHS Grampian, Grampian, UK; ${ }^{10}$ NHS Greater Glasgow and Clyde, Glasgow, UK; ${ }^{11}$ Walsall Healthcare NHS Trust, Walsall, UK; ${ }^{12}$ Newcastle upon Tyne Hospitals NHS Foundation Trust, Newcastle, UK;

${ }^{13}$ University of Southampton, Southampton, UK
}

Introduction The nutritional compositions of infant foods for special medical purposes (iFSMPs) are governed by the EU, and new regulations $(2016 / 127 ; 2016 / 128)$ were implemented to ensure standardisation and implementation of latest nutritional recommendations, scheduled to take effect by February 2020. Amongst the changes required, nutrient minimum and maximum levels were redefined, as well as mandatory supplementation of docosahexaenoic acid (DHA). Anecdotal evidence from clinical practice suggests that changes to formulations, minor or otherwise, may affect tolerance and acceptance in infants taking iFSMPs, especially those with complex medical conditions and backgrounds. A case-study series was conducted to evaluate iFSMPs reformulated by Nutricia Ltd to understand any possible impact on patient care.

Aim A multi-centre case-study series was conducted in infants and children who took iFSMPs manufactured by Nutricia Ltd for a range of clinical conditions. Gastro-intestinal tolerance, acceptance and compliance were evaluated over 28 days in each case-study.

Methods From 17 paediatric centres across the UK, 44 infants and children were recruited [mean age $16.5 \mathrm{~m}$; range 1.5-87], receiving one of the following iFSMPs prescribed for nutrition support relevant to their clinical condition: Infatrini $(n=9)$, Infatrini Peptisorb $(n=3)$, Neocate LCP $(n=9)$, Neocate Syneo $(n=1)$, Kindergen $(n=4)$, Monogen $(n=5)$, Energivit $(n=4)$, Locasol $(n=4)$, Galactomin $19 \quad(n=1)$, PKU Anamix Infant $(\mathrm{n}=4)$. Mean intake of baseline iFSMP was $683 \pm 275 \mathrm{ml}$ (which met $97 \%$ of prescribed daily volume), of which $n=16$ administered iFSMPs via enteral feeding tubes (the remaining orally). The managing Dietitian determined the prescribed daily volume of the reformulated iFSMP. Medical history was recorded at baseline, and growth, gastrointestinal tolerance, compliance and acceptance was measured at baseline and end of case-study.

Results Forty patients completed the 28-day evaluation $(n=4$ did not due to medical and other reasons, days on case-study ranged between 1-17). Gastrointestinal tolerance remained stable in the majority of case studies $(n=41$ including $n=1$ drop out), and any deviations were not attributed to the reformulated iFSMPs. For the patients that completed the 28-day evaluation, compliance remained stable $(n=33)$, and any reduction was related to increased complementary feeding or medical reasons. Mean intake of reformulated iFSMP was $579 \pm 254$ $\mathrm{ml}$ (which met 91\% of prescribed daily volume), where the majority of patients directly transitioned onto the reformulation $(n=41)$. No deterioration in medical conditions or growth were reported as a result of using the reformulated iFSMPs during any of the case studies. Furthermore, caregiver and HCP satisfaction was positively recorded in $89 \%$ of case studies.

Conclusion This multi-centre, case-study series demonstrates that the minor reformulation of iFSMPs manufactured by Nutricia Ltd in line with the Commission Delegated Regulations $(2016 / 127 ; 2016 / 128)$ to amend nutrient levels and include DHA are well tolerated, accepted and complied with in infants and children with various medical backgrounds. Furthermore, the reformulated iFSMPs continued to support growth and achieved positive caregiver and HCP satisfaction which is paramount to patient care. The reformulated iFSMPs used in this case study series have since been implemented into clinical practice in the UK, with support from Nutricia Ltd, and are now widely accepted.

10.1136/flgastro-2021-bspghan.40 